\title{
Attitude and readiness of Dentistry students in Kerman University of Medical Sciences concerning Tele-dentistry in 2017
}

Type of article: conference abstract

\author{
Fatemeh Tabatabaei ${ }^{1}$, Maryam Eslami Jahromi ${ }^{1}$, Kambiz Bahaadinbeigi ${ }^{2}$ \\ 1: Master Student of Health Information Technology, Faculty of Management and \\ Medical Information Sciences, Kerman University of Medical Sciences, Kerman, Iran. \\ 2: Associate Professor, Medical Informatics Research Center, Institute for Future Studies \\ in Health, Kerman. University of Medical Sciences, Kerman, Iran. \\ kambizb321@gmail.com
}

\begin{abstract}
Introduction: Tele-dentistry is the use of information and telecommunications technology for dental care, counseling, education and public awareness in the same way as remote medical. Teledentistry is a relatively new field that can change the dental care system. However, many dentists are unaware of the goals, benefits, and how to participate. Therefore, this study was conducted to determine the knowledge and attitude of dental students regarding telemedicine.

Methods: The present study is descriptive-analytic. The study population consisted of all dental students in Kerman in year 2017 and sample of 230 students selected by proportional sampling. For data collection, a complete English translation of an English-language article in the Journal of Clinical and Diagnostic Research, whose validity was confirmed by three of the leading medical informatics specialists in English, was then used to analyze the data using The software was SPSS 19.

Results: The findings of this study showed that there is no significant difference between the students' age, sex and term of education with their knowledge of tele-dentistry $(\mathrm{P}>0.05)$. Knowledge and attitude of $72 \%$ of students in tele-dentistry is very low. $40 \%$ of them said teledentistry would be very beneficial and more than half of them would be willing to do tele-dentistry in the future. $81 \%$ of them agreed that tele-dentistry could be a good tool for health education and oral health, and effective in educating the health of a large number of people. $75 \%$ of them felt that distance dental care saves time, and $81 \%$ of students believed that tele-dentistry was not financially feasible.

Conclusion: The results of this study showed that many respondents lacked adequate knowledge and knowledge about tele-dentistry. Hence, there is a huge need for awareness among dentists to further the future of dentistry in the future. Therefore, it is suggested that the related institutions take more serious measures for awareness and education about tele-dentistry for students.
\end{abstract}

KEYWORDS: Awareness, Dentistry Students, Tele-dentistry, Attitude

\section{Declaration of conflicts}

This abstract is selected from the First International Congress of Diseases and Health Outcomes Registry and First National Congress of Medical Informatics, 14-17 February 2017, Mashhad, Iran

\section{Authors' biography}

Kambiz Bahaadinbeigi, Associate Professor, Medical Informatics Research Center, Institute for Futures Studies in Health, Kerman. University of Medical Sciences, Kerman, Iran.Haft-bagh Highway, Kerman, Iran, PO Box: 7616913555Tel: +98.3431325406, Fax: +98.3431325221, E-mail: kambizb321@gmail.com 
Medical Technologies Journal, Volume: 1, Issue: 4, October-December 2017, Pages:76-136. Doi : https://doi.org/10.26415/2572-004X-vol1iss4

\section{References}

No references 